\title{
A DISINTEGRIN AND METALLOPROTEASE 33 POLYMORPHISMS AND LUNG FUNCTION DECLINE IN THE GENERAL POPULATION
}

Cleo C. van Diemen*, Dirkje S. Postma ${ }^{\#}$, Judith M. Vonk*, Marcel Bruinenberg", Jan P. Schouten* and H. Marike Boezen*

*Dept of Epidemiology, University of Groningen, University Medical Center Groningen, Groningen, The Netherlands

${ }^{\#}$ Dept of Pulmonology, University Medical Center Groningen, University of Groningen, Groningen, The Netherlands

"Dept of Medical Biology, University Medical Center Groningen, University of Groningen, Groningen, The Netherlands

WINNING ABSTRACT: ADAM33 (A Disintegrin and Metalloprotease 33) has been identified as a susceptibility gene for asthma and single nucleotide polymorphisms (SNPs) in this gene have been associated with excess decline of lung function in asthmatics.

To assess whether SNPs in ADAM33 are associated with accelerated lung function loss in the general population and with chronic obstructive pulmonary disease (COPD).

We have collected DNA from subjects of the Vlagtwedde/Vlaardingen cohort participating in the last survey in 1989/1990 after a follow up of 25 years. Information was collected every 3 years, including lung function measurements. We defined COPD as GOLD stage 2 or higher at the last survey. 1390 subjects from the cohort were genotyped for the following SNPs in ADAM33: $\mathrm{F}+1, \mathrm{Q}-1, S_{-} 1, S_{-} 2, T_{-} 1, T_{2} 2, V_{-} 4, S_{1}+5$. Differences in prevalence of SNPs were analyzed with chi-square tests. Linear mixed effects models were used to analyze $\mathrm{FEV}_{1}$ decline according to genotype.

In the whole population mean adjusted decline was 18.7 and $12.7 \mathrm{ml} \cdot \mathrm{y}^{-1}$ in females and males respectively. Individuals homozygous for minor alleles of SNPS S 2 and Q-1 and heterozygous for SNP S 1 had a significantly accelerated decline in $\mathrm{FEV}_{1}$ of respectively $4.9,9.6$ and $3.6 \mathrm{ml} \cdot \mathrm{y}^{-1}$ compared with wild type. We found a significantly higher prevalence of $\mathrm{SNPs} F+1$, S_1, S_2 and T_2 in subjects with COPD.

We demonstrated that SNPs in ADAM33 are associated with accelerated lung function decline in the general population. These SNPs are also risk factors for COPD.

\section{POLYMORPHISMS IN SURFACTANT PROTEINS ARE ASSOCIATED WITH FEV1 DECLINE AND DEVELOPMENT OF COPD IN THE GENERAL POPULATION}

Cleo C. van Diemen*, Dirkje S. Postma\#, Judith M. Vonk* and H. Marike Boezen*

*Dept of Epidemiology, University of Groningen, University Medical Center Groningen, Groningen, The Netherlands

\#Dept of Pulmonology, University Medical Center Groningen, University of Groningen, Groningen, The Netherlands

WINNING ABSTRACT: Surfactant proteins (SP) are involved in the first response to microorganisms in the lung, regulation of inflammation and structure of alveoli. Single nucleotide polymorphisms (SNPs) in these genes have been associated with COPD prevalence.

To assess whether SNPs in SP-A1, $-A 2,-B$, and $-D$ are associated with development of COPD and FEV decline in the general population.

1390 Caucasians from a longitudinal follow-up study (characteristics median (range): $51 \%$ male, pack-years 8 (0-161), age 52 (35-79) years, FEV 92 (15-137) \%pred). COPD was defined as GOLD $\geqslant$ stage II. We analyzed: SP-A1 Val19Ala, Leu50Val, Arg219Trp; SP-A2 Asn9Thr, Pro91Ala, Ser140Ser; SP-B Ile131Thr; SP-D Met11Thr, Thr160Ala; haplotypes of SP-A1 and -A2. Differences in prevalence of genotypes were analyzed with $\mathrm{X}^{2}$-tests. $\mathrm{FEV}_{1}$ decline according to genotype was analyzed using linear mixed effect models with adjustment for confounders.

Heterozygous subjects for the SP-D Met11Thr SNP had a significantly larger decline in $\mathrm{FEV}_{1}$ of $2.9 \mathrm{ml} \cdot \mathrm{y}^{-1} \mathrm{compared} \mathrm{to} \mathrm{wild}$ type $(P=0.03)$. In COPD subjects, those heterozygous for SNP SP-A1 Leu50Val had $11.0 \mathrm{ml}^{-1} \mathrm{y}^{-1}$ less $\mathrm{FEV}_{1} \mathrm{decline} \mathrm{compared}$ to wild type $(p=0.018)$. SNPs SP-D Met11Thr, SP-A1 Leu50Val, and SP-A2 Ala91Pro were differently distributed in subjects with and without COPD (P-values respectively 0.045, 0.002, 0.024).

This is the first study to show that the SP-D Met11Thr SNP is associated with faster decline in FEV and $_{1}$ with development of

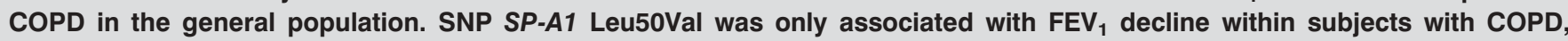
indicating that SNPs may have different effects depending on disease state. 


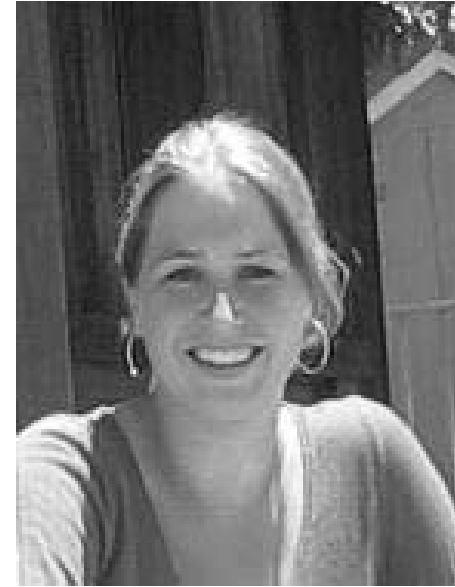

Cleo C. van Diemen

Dept of Epidemiology, University of Groningen, University Medical Center, Groningen, The Netherlands

\section{MY JOB AND THE UNIT IN WHICH I WORK}

I am a PhD student at the Dept of Epidemiology in the University Medical Center Groningen (UMCG; Groningen, the Netherlands). This department has a long-standing history in the epidemiology of pulmonary diseases, with a specific focus on risk factors for the development and progression of asthma, allergy and chronic obstructive pulmonary disease (COPD).

Nowadays, a large part of the research is specifically focused on the role of genetics and gene-environment interaction in the development of respiratory disease. In addition to this specific line of research, the Dept of Epidemiology offers statistical and methodological advice to other research groups of the UMCG, and scientific and methodological training to $\mathrm{MD}$ and $\mathrm{PhD}$ students of the UMCG.

\section{MY WINNING ABSTRACT AS PART OF MY RESEARCH}

My PhD project is entitled "Genetic contribution to the course of lung function and pathophysiology of enhanced lung function loss in a large cohort followed up for 30 years", and is funded by the Dutch Asthma Foundation (principal investigators: Dr H.M. Boezen, Dept of Epidemiology, and Prof. Dr D.S. Postma, Dept of Pulmonology, UMCG). In this project, I use data from the Vlagtwedde/Vlaardingen cohort, which comprises Caucasian subjects from the general population of the Netherlands, who have been followed from 19651990. Every 3 yrs, lung function measurements and bronchial hyperresponsiveness tests were performed, and questionnaires were collected. We have isolated DNA from 2,467 subjects at the last survey. Since lung function measurements were registered every 3 yrs, we can accurately model the effects of genetic mutations on the course of lung function over time, and on the development of COPD.

COPD is almost completely caused by the environmental factor smoking, with the exception of the genetic predominance of $\alpha_{1}$-antitrypsin (AT) deficiency gene, the carriers of which need no further environmental smoke exposure to develop a phenotypic expression of COPD. However, $\alpha_{1}$-AT deficiency accounts for only a small number of COPD cases worldwide $(<1 \%)$ and therefore cannot explain the majority of individuals who develop COPD at elderly age ("the garden variety $\left.\mathrm{COPD}^{\prime \prime}\right)$.

COPD is characterised by reduced levels of forced expiratory volume in one second (FEV1), which provides the most important and robust phenotype of COPD, and predicts the progression of the disease and overall mortality $[1,2]$. The level of lung function is both environmentally and genetically determined. In past decades, the environmental determinants have been studied extensively, i.e. smoking, air pollution, occupational exposure, diet and exposure to respiratory allergens. These studies have shown clear associations between certain exposures, e.g. cigarette smoking, and the development of COPD. However, it is less clear why some smokers develop COPD and other smokers do not. It is important to understand why some people are genetically susceptible to developing COPD while others are not, since it may provide new insights into the disease, potentially leading to better treatments. The genetic determinants have been studied less frequently and consistently, partly because they are more complicated to study, as COPD is a disease that is merely expressed at older ages. The "traditional" types of genetic studies, like those performed within families, are difficult to perform because the parents of individuals with COPD have often already died and the children of subjects with COPD are likely to be too young to manifest airway obstruction at that age.

Case-control studies investigating COPD patients and healthy controls are generally used to study the genetics of COPD. So far these studies have been small in size. Furthermore, the disease is only investigated when symptoms are present, without acknowledging the different patterns of lung function loss that evolve, i.e. abnormally high rate of decline in lung function, submaximally attained level of lung function, or abnormally early age of onset of decline. Genetic factors may affect any or a combination of these different patterns of lung function loss. Therefore, it is important to study multiple measurements of lung function in a well-characterised cohort followed throughout life. We have the unique opportunity to investigate susceptibility genes for excess lung function decline in the Vlagtwedde/Vlaardingen cohort. This large cohort of male and female smokers and nonsmokers from a general population sample has been followed over a 25 -yr period. This will not only allow us to study the contribution of genetic variations to decline in lung function, but also to study the genes involved in previously well-established risk factors for lung function course and decline in this population, i.e. airway hyperresponsiveness and blood eosinophilia.

In our studies, we have chosen to study a robust phenotype, namely level of FEV1 and rate of decline in FEV1. The publication that was awarded best paper (third prize) in the Occupation and Epidemiology assembly describes the results obtained in a study in which we analysed the effects of single nucleotide polymorphisms (SNPs) in ADAM33 on FEV1 decline and the development of COPD in the Vlagtwedde/ Vlaardingen population [3]. We found that SNPs in ADAM33 are predictive of excess FEV1 decline in this general population and development of COPD. Combined with the previous findings that the ADAM33 gene is associated with FEV1 decline in asthmatics [4], these results suggest that SNPs in $A D A M 33$ affect lung function decline overall, independently of 
asthma or COPD status, and may thus constitute a lung function ageing gene.

The abstract belonging to the best poster award describes the association of polymorphisms in surfactant proteins-A1, -A2, $-\mathrm{C}$ and $-\mathrm{D}$ with development of COPD and FEV1 decline in the Vlagtwedde/Vlaardingen population.

\section{MY RESEARCH AS PART OF MY WORKING GROUP/ RESEARCH TEAM}

My research fits within the Dept of Epidemiology's research line that focuses on the role of genetics and gene-environment interaction in the development of respiratory disease, specifically COPD. This research line is embedded within the Groningen Research Institute on Asthma and COPD (GRIAC), which is part of the governmentally accredited organisation GUIDE (Groningen University Institute for Drug Exploration, Groningen, The Netherlands). There is an intensive collaboration between researchers of the GRIAC, consisting of members from different disciplines: allergology, general practice, molecular pharmacology, pathology, paediatric pulmonology, pulmonology, respiratory insufficiency and epidemiology. Additionally, collaboration exists with the departments of dermatology, gastroenterology, genetics, haematology, medical oncology and transplantation. The central goal of the research of the GRIAC is to translate fundamental findings to the clinical situation and vice versa. To this end, the GRIAC focuses on the following core topics related to asthma and COPD: 1) epidemiological risk factors; 2) genetic background; 3) pathophysiology and pathogenesis of allergen-, environment- and smoke-induced disease; and 4) assessment, modulation and intervention in disease severity and progression.
The PhD project that I perform fits into the first and second aims of the GRIAC, namely identifying genes associated with COPD and lung function decline regarded in an epidemiological background.

\section{THE IMPACT OF MY WORK ON CLINICAL OR RESEARCH PRACTICE}

My study will identify genetic risk factors for development of COPD, excess lung function decline and/or impaired lung function. Since it is still unclear why some smokers do and some smokers do not develop COPD, this may provide new insights into the pathophysiology of COPD, which is, to the present date, not fully understood. Furthermore, it may provide new insights in treatments for COPD, a disease that is still not properly managed.

\section{REFERENCES}

1 Ekberg-Aronsson M, Pehrsson K, Nilsson JA, Nilsson PM, Lofdahl CG. Mortality in GOLD stages of COPD and its dependence on symptoms of chronic bronchitis. Respir Res 2005; 6: 98.

2 Sin DD, Man SF. Chronic obstructive pulmonary disease as a risk factor for cardiovascular morbidity and mortality. Proc Am Thorac Soc 2005; 2: 8-11.

3 van Diemen CC, Postma DS, Vonk JM, Bruinenberg M, Schouten JP, Boezen HM. A disintegrin and metalloprotease 33 polymorphisms and lung function decline in the general population. Am J Respir Crit Care Med 2005; 172: 329-333.

4 Jongepier H, Boezen HM, Dijkstra A, et al. Polymorphisms of the ADAM33 gene are associated with accelerated lung function decline in asthma. Clin Exp Allergy 2004; 34: 757-760. 